\title{
Proteus Mirabilis Septicemia and Meningitis in a Neonate
}

\author{
Emma Archibong Omoruyi ${ }^{\mathrm{a}}$, Monaliza Evangelista ${ }^{\mathrm{a}, \mathrm{b}}$
}

\begin{abstract}
A 6-day-old premature baby boy presented in a pediatric clinic for severe jaundice, poor feeding and lethargy. He was born to a 24-year-old mother who was primigravida. Pregnancy was complicated by gestational diabetes, appropriately treated chlamydial infection, oligohydramnios and inadequate prenatal care. On physical examination, he was hypotonic and irritable with significant jaundice seen from his head to his lower extremities. Transcutaneous bilirubin obtained in clinic showed a level of $14 \mathrm{mg} / \mathrm{dL}$. A serum bilirubin level was immediately ordered and it confirmed an elevated unconjugated bilirubin level of $18.9 \mathrm{mg} / \mathrm{dL}$. He was admitted to the inpatient unit and a full septic work-up was initiated. Appropriate antibiotics were administered to cover for the most common pathogen involved in neonatal sepsis. A few hours into the admission, his condition deteriorated requiring intubation for multiple consecutive apneic episodes and seizure-like activity. Initial laboratory evaluation included an abnormal white blood cell count of $2,600 / \mu \mathrm{L}$ with left shift, elevated lactic acid of $9 \mathrm{mmol} / \mathrm{L}$ and a low bicarbonate level of $16 \mathrm{mmol} / \mathrm{L}$ further confirming acidosis. Analysis of cerebrospinal fluid revealed a white blood cell count of $>300 / \mu \mathrm{L}$, low glucose with a level of $7 \mathrm{mg} / \mathrm{dL}$ and elevated protein level of $645 \mathrm{mg} / \mathrm{dL}$. Due to his rapid clinical deterioration a few hours into the admission, a computed tomography scan of the brain was obtained. Computed tomography scan of brain showed significant damage to brain parenchyma consistent with anoxic brain injury. He expired on his second day of admission. Proteus mirabilis was the causative agent.
\end{abstract}

Keywords: Proteus mirabilis; Neonatal meningitis; Neonatal sepsis

Manuscript accepted for publication April 26, 2013

${ }^{\mathrm{a}}$ Department of Pediatrics, University of Texas Health Science Center at Houston, 6431 Fannin Street, JJL-495, Houston, Texas 77030, USA

${ }^{\mathrm{b}}$ Corresponding author: Monaliza Evangelista, Department of

Pediatrics, University of Texas Health Science Center at Houston,

6431 Fannin Street, JJL-495, Houston, Texas 77030, USA.

Email: monaliza.s.evangelista@uth.tmc.edu

doi: http://dx.doi.org/10.14740/jmc1290w

\section{Introduction}

Proteus mirabilis is an uncommon infectious organism in neonatal sepsis. To our knowledge, there have been only a limited number of cases that have been described in literature worldwide that implicates Proteus as a cause of infection involving neonatal meningitis and septicemia. This organism is a common inhabitant of the human intestinal tract and most cases of Proteus infection in humans are considered community acquired infections [1]. In neonates, Proteus infection accounts for a very small percentage of neonatal sepsis. A review by Unhanand et al of bacterial meningitis cases admitted in Children's Medical Center in Dallas from period 1969 to 1989 involving gram-negative enteric bacillary meningitis in neonates and infants identified 98 cases and $4 \%$ of those were caused by Proteus mirabilis [2]. Route of infection has not been established but presumed to be ascending infection during delivery due to its early presentation. In most of the cases reported in literature, the course of the infection appears to be fatal involving severe neurological complication with cerebral abscess and pneumocephalus formation despite institution of appropriate antibiotics [2-5].

\section{Case Report}

A 6-day-old premature baby boy was brought into pediatric clinic with a 2-day history of poor feeding, lethargy and worsening jaundice. He was born at 36 weeks gestation and was discharged at 2 days of life after an uneventful stay in the newborn nursery. Per hospital protocol, he followed up in clinic early due to prematurity and jaundice. There was no history of injury. His mother was a 24-year-old primigravida and received inadequate prenatal care. Her pregnancy was complicated by gestational diabetes, chlamydial infection that was appropriately treated and oligohydramnios. His mother also had urinary tract infection caused by E. coli during her pregnancy and she received proper treatment. Repeat urine cultures on subsequent prenatal visit did not grow any bacteria. Maternal laboratory screenings for HIV, Group B Streptococcus, syphilis and hepatitis B were all negative. In 
clinic, the baby appears irritable on exam with significant jaundice from the head to his lower extremities. He was hypotonic and appeared disinterested with a feeding given in clinic. Transcutaneous bilirubin was performed and a level of $14 \mathrm{mg} / \mathrm{dL}$ was obtained. A serum bilirubin was immediately sent and he was admitted for management of jaundice with concern for dehydration and possibly sepsis.

Immediately after admission, he had brief episode of head and right upper extremity rigidity. He also failed to react appropriately to painful stimuli with placement of intravenous access and lumbar puncture. A full septic work-up was performed and intravenous antibiotics with ampicillin and cefotaxime was given. Three hours into admission, his condition deteriorated. He had multiple apneic episodes observed by the hospital staff along with a seizure-like activity. He was intubated and transferred to the intensive care unit (ICU). He also developed hypotension and was placed on vasopressor support.

Immediate laboratory investigation showed unconjugated hyperbilirubinemia with a serum level of $18.9 \mathrm{mg} / \mathrm{dL}$. His white blood cell count was $2,600 / \mu \mathrm{L}$ with seven bands and $22 \%$ neutrophils. Serum electrolyte abnormalities were significant for low bicarbonate level. Lactic acid was elevated at a level of $9.0 \mathrm{mmol} / \mathrm{L}$. Cerebrospinal fluid (CSF) microscopy showed a white cell count of $>300 / \mu \mathrm{L}$ (differential was not performed due to WBC degenerated per lab personnel), a CSF glucose of $7 \mathrm{mg} / \mathrm{dL}$ and protein of $645 \mathrm{mg} / \mathrm{dL}$.

A brain computed tomography immediately performed after transfer to ICU showed global anoxic injury involving the entirety of the brain with effacement of the basal cisterns consistent with superior inferior transtentorial herniation and subarachnoid hemorrhage.

Early into the second day of admission, his blood and CSF culture grew gram-negative bacilli and was later identified as Proteus mirabilis. The organism appears to be sensitive to the antibiotics administered but his condition continued to deteriorate. Due to cardiovascular collapse associated with sepsis, he continued to need multiple vasopressor support. His lactic acid remained elevated. Coagulation profiles were deranged and he required transfusion of blood products for correction. He was removed from sedation for several hours but his physical examination did not reveal any spontaneous movements. His pupils were non-reactive and he did not have any spontaneous respiratory effort observed with ventilator support.

Electroencephalogram performed revealed severe suppression of brain activity consistent with anoxic brain injury. His parents were informed of the poor prognosis of his condition from severe brain damage and continued overwhelming sepsis and shock. After multiple family meetings, his parents decided to withdraw support. He was extubated later into the second day of admission and he died a few minutes after. Autopsy was offered and performed. Results demonstrated severe autolysis and maceration of the brain. There is severe subarachnoid hemorrhage at the base of the brain and subdural hematomas over the tentorium cerebelli.

\section{Discussion}

Proteus mirabilis is not a commonly isolated organism in cases of neonatal sepsis and infections of the central nervous system. Some report it as the causative agent in about $4 \%$ of cases of neonatal meningitis [3]. Cerebral abscess formation and pneumocephalus has been described as also being associated with Proteus infections [2-5]. Our patient had brain findings that were most likely caused by the overwhelming sepsis.

Clinical features appeared as early as day 6 of life, and with no evidence of ear, sinus or urinary infections, ascending maternal transmission is the most likely route of infection. Reiner et al reported four fatal cases of Proteus infection associated with a maternal Proteus urinary tract infection [6]. This was the first report that possibly linked maternal infection to development of neonatal infection. Given the poor outcomes of most cases reported in the literature in the past four decades, early diagnosis method may be warranted through antepartum screening with appropriate institution of prophylactic antibiotics during delivery. Though the case reports involving Proteus have been sporadic, there is clear evidence to show the infection is fatal in its early course. We hope that with increased awareness, an evaluation of the cost-effectiveness associated with implementing a screening can be examined. Similar discussions in the obstetric community are taking place regarding universal toxoplasmosis screening [7]. We think Proteus mirabilis should be added to the discussion given its similar association with devastating outcomes.

Although infection of this organism is not well reported and its true prevalence is unknown, the gravity of the illness should still raise awareness. Pediatricians should have a high index of suspicion when dealing with cases of meningitis and septicemia that shows rapid deterioration of clinical condition despite of appropriate management of antibiotics. Moreover, better surveillance data need to be gathered to better see the magnitude of the infection.

With this case report, we continue to expand the causative agents associated with early neonatal sepsis and the organisms association with abscess formation. This report provides further evidence that neonatal infection with Proteus mirabilis is typically fatal and early screening methods should be investigated and implemented.

\section{References}

1. Feigin R, Cherry J, Demmler G, Kaplan, S. Textbook of 
Pediatric Infectious Disease. 5th ed. Philadelphia: WB Saunders; 2003.

2. Smith ML, Mellor D. Proteus mirabilis meningitis and cerebral abscess in the newborn period. Arch Dis Child. 1980;55(4):308-310.

3. Unhanand M, Mustafa MM, McCracken GH, Jr., Nelson JD. Gram-negative enteric bacillary meningitis: a twenty-one-year experience. J Pediatr. 1993;122(1):15-21.

4. Phan H, Lehman D. Cerebral abscess complicating Proteus mirabilis meningitis in a newborn infant. J Child Neurol. 2012;27(3):405-407.

5. Kassim Z, Aziz AA, Haque QM, Cheung HA. Isolation of Proteus mirabilis from severe neonatal sepsis and central nervous system infection with extensive pneumocephalus. Eur J Pediatr. 2003;162(9):644-645.

6. Renier D, Flandin C, Hirsch E, Hirsch JF. Brain abscesses in neonates. A study of 30 cases. J Neurosurg. 1988;69(6):877-882.

7. Boyer KM, Holfels E, Roizen N, Swisher C, Mack D, Remington J, Withers S, et al. Risk factors for Toxoplasma gondii infection in mothers of infants with congenital toxoplasmosis: Implications for prenatal management and screening. Am J Obstet Gynecol. 2005;192(2):564571. 